\title{
The cemented twin-peg Oxford partial knee replacement survivorship: A cohort study
}

\author{
Stephen H. White ${ }^{\text {a,*,1 }}$, Sharon Roberts ${ }^{\text {a }}$, Jan Herman Kuiper ${ }^{\text {a,b }}$ \\ a The Robert Jones and Agnes Hunt Orthopaedic Hospital NHS Foundation Trust, Oswestry, Shropshire SY10 7AG, United Kingdom \\ ${ }^{\mathrm{b}}$ Institute for Science and Technology in Medicine, Keele University, United Kingdom
}

\section{A R T I C L E I N F O}

\section{Article history:}

Received 31 October 2014

Received in revised form 9 February 2015

Accepted 16 March 2015

Available online $\mathrm{xxxx}$

\section{Keywords:}

Oxford twin-peg cemented knee replacement

Unicompartmental knee replacement

Anteromedial osteoarthritis

Survival analysis

Patient reported outcome measures (PROMs)

\begin{abstract}
A B S T R A C T
Background: A new twin-peg version of the Oxford knee was introduced in 2003. However, until now there has been no information about its survivorship. The aim of this study was to determine the survivorship, and the patients' perception of outcome over time.

Methods: A cohort of all patients treated from 2003 until 2009 using the twin-peg Oxford partial knee was contacted. The main indication for treatment was anteromedial osteoarthritis (AMOA). The Oxford Knee Score (OKS), American Knee Society Functional (AKS-F) score and satisfaction rate were obtained, and the time-tofailure was used to perform a survival analysis.

Results: There were 249 patients treated, with 288 medial cemented implants. Of these, 248 patients with 287 implants could be contacted and implant survival or failure was verified. Their mean age was 67 years (range: 34-94). The mean follow-up time was 5.1 years (maximum: 9.2). The nine years cumulative implant survival rate for all cases using revision for any reason to define failure was $98 \%$ ( $95 \% \mathrm{CI}, 84$ to 100 ). There were no cases of femoral loosening. The mean OKS was 22 pre-operatively, 41 at two years, and 41 at final review, at which point $96 \%$ of patients were very or fairly pleased with the result.

Conclusion: The survivorship of the twin-peg knee was better than that of the single peg knee at our centre, and appeared no worse than the results of the single peg knee at the originating centre. It can offer secure femoral fixation, sustained clinical benefit and patient satisfaction.

Level of evidence: Level IV case-series.
\end{abstract}

(c) 2015 Elsevier B.V. All rights reserved.

\section{Introduction}

Surgeons who implant unicompartmental knee replacements face a challenge to consistently achieve excellent long-term results in terms of a full range of movement, freedom from pain and long-term survivorship. Throughout the world there have been mixed results with a variety of modes of failure [1,2]. Although there was no case of femoral and only one case of tibial loosening in a series of 1000 cases of patients treated using the Oxford knee at the originating centre [1], others found loosening to be the most common cause of failure [3,4]. In a series of 1165 operations carried out between 1996 and 2008, loosening accounted for $40 \%$ of 89 revisions [3]. The National Joint Registry for England, Wales and Northern Ireland Annual Report of 2013 reports loosening to be the commonest single cause of failure of mobile bearing unicompartmental knee replacements, responsible for $25 \%$ of all revisions [4]. Early femoral loosening proved to be an issue in our practice

\footnotetext{
* Corresponding author.

E-mail addresses: Stephen.White@rjah.nhs.uk (S.H. White),

Sharon.Roberts@rjah.nhs.uk (S. Roberts), jan.kuiper@nhs.net (J.H. Kuiper).

1 Also at the Nuffield Health Hospital, Longden Road, Shrewsbury, SY3 9DP, United Kingdom.
}

as well, at a rate of three of 31 cases within three years [5] and one of 78 knees by two years [6]. For this reason, we were keen to use a more securely fixed version of the Oxford implant.

In 2003, the twin-peg Oxford femoral component was made available. It had been primarily designed to allow over $165^{\circ}$ of flexion after unicompartmental replacement. Whilst the natural human knee can accommodate such flexion in sitting and kneeling, the single peg Oxford femoral component would only be in partial contact with the bearing at such extremes of flexion (Fig. 1). The resulting increased stresses and edge contact could accelerate polyethylene wear. The new femoral component incorporating the extra femoral peg increases the arc and can be inserted in greater flexion, thereby adding more contact with the bearing in deep flexion. With the approval of our new procedure committee and our patients' informed consent, we decided to discontinue the phase III single peg Oxford partial knee in 2003 in favour of the twin-peg version (Fig. 2). In 2012 we reported our clinical results of the first 100 patients who had all reached two years of follow-up, and showed a mean Oxford Knee Score (OKS) of 41, a mean American Knee Society knee (AKS-K) score of 93, a functional (AKS-F) score of 84 and a mean $130^{\circ}$ range of flexion [7]. The radiological analysis at two years showed no evidence of femoral loosening [7]. However, the clinical results and survivorship of this implant beyond two years are not known. 

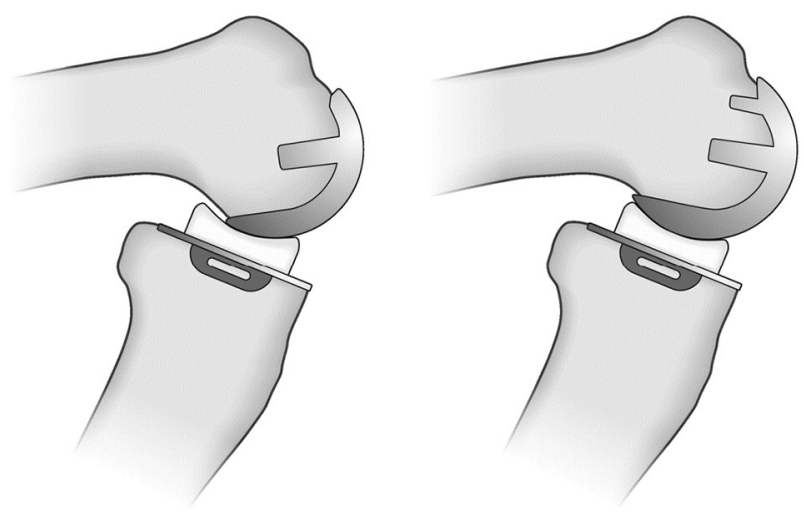

Fig. 1. Single peg Oxford knee on the left, twin peg version on the right.

The aim of the present study therefore is to report the longer-term implant survivorship and clinical outcome of the cemented twin-peg Oxford partial knee in a patient cohort which includes the original group of 100 patients, treated between 2003 and 2005, plus those treated since until the end of 2009.

\section{Patients and methods}

\subsection{Patients and outcome measures}

This study comprised all patients operated upon using the cemented twin-peg Oxford partial knee (Biomet UK Ltd, Bridgend, UK) by the senior author or under his direct supervision up to and including 2009. Patients had been selected for treatment if they had medial compartment osteoarthritis, which in most cases meant anteromedial osteoarthritis [8], but also included patients who had previous trauma or avascular necrosis. In AMOA there should be full thickness cartilage loss on both sides of the medial compartment with bone on bone contact. There should also be preservation of full thickness cartilage in the lateral compartment. The medial collateral ligament should be functionally normal as demonstrated by a correctable intra-articular varus deformity at $20^{\circ}$ of flexion. The anterior cruciate ligament (ACL) should be normal as inspected and probed at surgery. The presence of a chondral ulcer on the inter-condylar margin of the lateral femoral condyle can be ignored as well as the patient's age, weight, level of activity and the presence of chondrocalcinosis. Patients were excluded if there was evidence of inflammatory arthritis or if there was fixed flexion of the knee beyond $10^{\circ}$. Patients with patellofemoral osteoarthritis, regardless of the degree

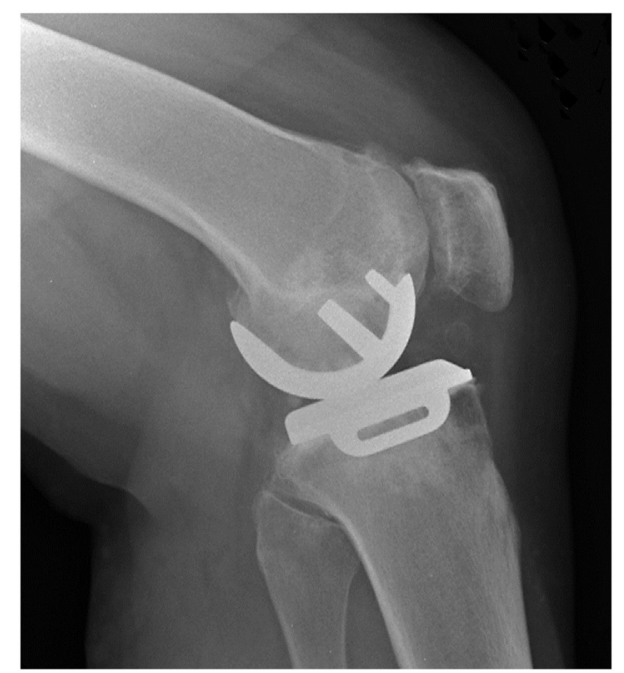

Fig. 2. Lateral radiograph showing the cemented twin peg Oxford knee. or pattern, were included because preservation of the cruciate ligament was considered more beneficial in terms of patellofemoral contact stresses compared to the pathomechanics that is inevitable on sacrificing the anterior cruciate ligament when carrying out total knee replacement.

All patients were followed up at two years with radiographs, clinical examination, and recording of the Oxford Knee Score (OKS) and American Knee Society Functional (AKS-F) score $[9,10]$. In addition, the range of motion of each knee was recorded by a research physiotherapist (SR). A satisfaction questionnaire and a form to record any complications that had occurred during the first two years were completed.

All patients who were alive and had the implant in-situ at the two years clinical follow-up were contacted again for final review up to the tenth year after surgery using a variety of techniques: post, phone call and ultimately by contacting the GP. They were assessed using a postal questionnaire which included the OKS, the AKS-F, the Tegner Activity score [11], and a satisfaction questionnaire as used in Oxford [1].

The process of final review was begun in March 2012 and the database finalised in July 2013. The extended duration was because of difficulties in some cases of contacting patients. For patients who were non-contactable, including those who had died, information was gathered from hospital notes and by contacting the general practitioner to confirm whether the knee had been revised or not.

The operative technique was as previously described [7].

\subsection{Statistical analysis}

A multilevel model with a random intercept was used to determine the difference between OKS scores pre-operatively and two years post-operatively and between OKS scores and AKS-F scores two-years post-operatively and at the latest follow-up. The multilevel method was used to properly account for scores obtained from patients with bilateral implants. It also allowed including all patients with at least one score to investigate changes in score over time.

Implant survival was determined using the life-table method and Greenwood estimates of the $95 \%$ confidence intervals [12]. The lower confidence limit took account of the effective sample size at each time point. Implant failure was defined as revision for any reason.

All statistical analyses were performed using R vs 3.0.2, using the packages "survival" and "nlme". All statistical tests were performed as two-tailed tests, and a p-value below 0.05 was assumed to denote statistical significance.

\section{Results}

\subsection{Patient demography}

There were 288 medial cemented implants, inserted in 249 patients (Table 1). There were 210 unilateral and 39 bilateral procedures. Only one bilateral case was simultaneous,

Table 1

Demographic details.

\begin{tabular}{lll}
\hline Characteristic & n or mean $(\mathrm{SD})$ & Range \\
\hline Number of patients & 249 & \\
Female & 121 & \\
Male & 128 & \\
Number of implants & 288 & 34 to 94 \\
Unilateral & 210 & \\
Bilateral & 39 & \\
$\quad$ Staged & 38 & \\
Simultaneous & 1 & \\
Age at operation & $67.0(9.8)$ & \\
Indications & 267 & \\
AMOA & 11 & \\
AMOA extended & 4 & \\
AVN/OCD & 3 & \\
Trauma & 3 & \\
Others &
\end{tabular}


the rest were staged. The mean age of the patients at the time of operation was 67 years (range: 34 to 94$)$. There were roughly equal numbers of women $(121 ; 48.6 \%)$ and men (128; 51.4\%; Table 1). All patients could be followed up, either directly or through their GP, except one patient who had flown in for the surgery from abroad. Therefore, the follow-up rate of the study was $99.6 \%$

\subsection{Indications for surgery}

The procedure was undertaken in 267 knees for AMOA and 21 knees for other indications (Table 1). Eleven patients had extended indications for AMOA, recorded at the time of surgery. In these cases either the ACL was abnormal or there was more extensive damage to other joint compartments, but the joint was still deemed suitable for the procedure. In addition, four knees demonstrated avascular avascular necrosis (AVN) or osteochondritis dissecans (OCD) and three knees medial compartment arthritis due to previous trauma, and one patient had a previous high tibial osteotomy. Because of the small numbers of patients with conditions other than AMOA these have been grouped together as "other indications".

\subsection{Follow-up period}

The mean follow-up period was 5.1 years, with a minimum of 2.7 years (excluding patients who died or whose implant failed) and a maximum of 9.2 years. For $74 \%$ of the knees, the follow-up period was at least four years.

\subsection{Revision and survival}

Five patients (five cases) required a revision: two patients who had AMOA indications and three patients who had another indication (Table 2). The commonest reason for revision was progression of osteoarthritis to the lateral compartment (three cases). In addition, two patients required a revision due to unexplained pain; one was revised to a total knee replacement with improved outcome, while the other patient was revised elsewhere and the outcome is unknown. All of the revisions were during the first five years after knee replacement.

There were no cases of dislocation of the bearing, no cases of femoral loosening, and no deep infections either early or late. One patient had complications of iatrogenic, complete medial collateral division during the tibial cut due to misplacement of the medial retractor. The ligament was repaired, but during a period in a varus cast the tibial plateau fractured. The fracture went on to heal with conservative treatment and although the patient was "very disappointed" it has not led to revision.

The nine years cumulative implant survival rate for all cases using revision for any reason as the definition of failure was $98 \%$ ( $95 \%$ CI, 84 to 100; Fig. 3 and Table 3 ). The nine years cumulative implant survival for AMOA cases was 99\% (95\% CI, 89 to 100) and the seven years cumulative implant survival for other indication cases was $82 \%$ (95\% CI, 55 to 100; Fig .2). Patients whose knees were treated for AMOA had a significantly better survivorship than patients with other indications (Fig 4; log rank test, $\mathrm{p}<0.001$ ).

\subsection{Clinical outcome}

The mean values for the OKS were 22.1 pre-operatively, 40.8 at two years, and 41.0 at final review (Table 4). Female patients scored a mean of 3.6 points lower than male patients, a significant difference ( $\mathrm{p}=0.001$ for all time points). The mean difference in scores was 19.0 points ( $95 \% \mathrm{Cl}, 17.4$ to 20.7 ) from pre-op to two-years follow-up and 0.22 points ( $95 \% \mathrm{CI},-0.69$ to 1.14 ) between two years and the latest follow-up, with no significant difference between the genders (Table 4). The mean AKS-F scores were 80.0 points at two years and 76.7 at the latest follow-up for female patients, and 88.2 and 83.9 points, respectively, for male patients with a significant difference between the genders and a significant drop over time (Table 4). Female patients had a mean Tegner Acivity score of 2.0 and a mean range of motion of $128^{\circ}$, whereas male patients had a mean Tegner Acivity score of 2.8 and a mean range of motion of $131^{\circ}$, a significant difference in both cases (Table 4). A higher age at operation significantly reduced the AKS-F and the Tegner Acivity score, but did not influence the OKS (Table 4). The indication (AMOA versus other) did not influence any of the scores (Table 4).

At final review 220 patients ( 255 knees) rated their satisfaction. Of these patients, 191 (87\%; 223 knees) were very pleased, 20 (nine percent; 20 knees) were fairly pleased, five (two percent, five knees) were not very pleased and five (two percent, seven knees) were very disappointed. The sum of patients was 221 rather than 220 because one bilateral patient rated one knee differently to the other.

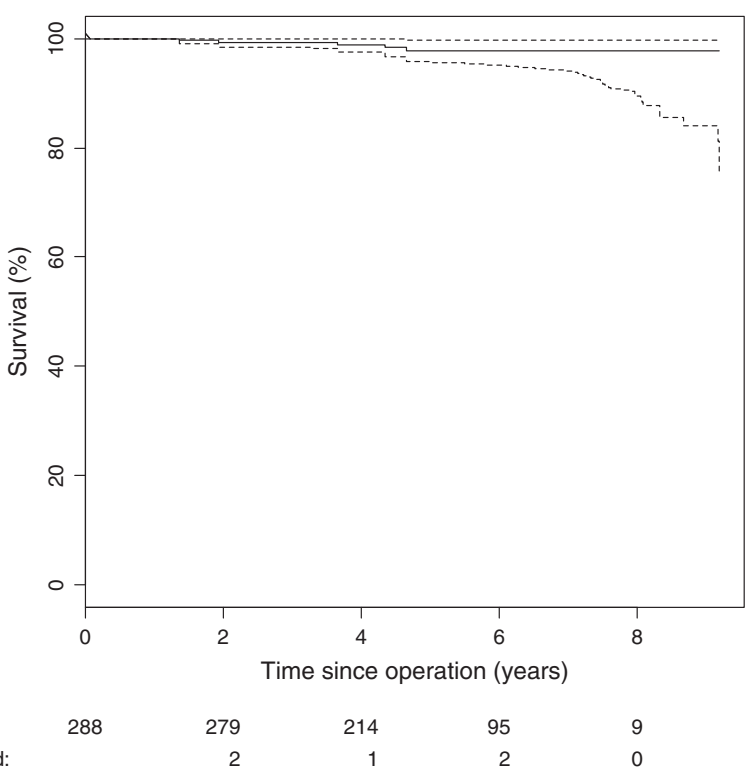

Fig. 3. Survival curve for unicondylar Oxford knee prosthesis. Dashed lines represent 95\% confidence interval, taking into account number currently at risk.

\section{Discussion}

This is the first study reporting survival of the cemented twin-peg Oxford medial unicompartmental knee replacement, showing a survival rate of $98 \%$ at nine years. This rate is comparable with the results using the single peg cemented implant from the originating centre reported by Pandit [1] and an independent centre reported by Svärd [2,13]. This outcome is better than our previous results using the cemented single peg implant [5,6] and the nine years survival rate of $89 \%$ reported in the National Joint Registry of England, Wales and Northern Ireland (NJR) or survival rates from other national joint registers [4,14]. The survival rate was highest when carried out for the patients who satisfied the original recommended indications for the Oxford knee: anteromedial osteoarthritis (AMOA) [8]. There were no cases of femoral loosening. Female and male patients had a similar improvement in clinical outcome, but female patients had lower preoperative as well as postoperative clinical scores and a lower postoperative range of motion. At the latest review, 96\% of patients were pleased with their knee replacement.

According to the 2013 report of the NJR, the cumulative percentage probability of revision of the Oxford unicompartmental knee replacement is $11.5 \%$ at the same time point of nine years [4]. Although the NJR does not distinguish between single-peg and twin-peg Oxford knees, the twin-peg version has not been widely available until after 2009 suggesting most of the Oxford knees recorded in the NJR are single peg versions. This rate of failure is typical of unicompartmental devices, with even higher failure rates recorded for the AMC/Uniglide (11.8\%) and Preservation (17.3\%) devices [4]. The results for the twin-peg implant described in the current study are therefore considerably better than those from the NJR, but what is the reason for this? Whilst it is our contention that the twin-peg has advantages over the single peg

Table 2

Overview of revision cases.

\begin{tabular}{|c|c|c|c|c|}
\hline Revision case & Time to revision & Indication & Reason for revision & Revision and outcome \\
\hline 1 & 1.4 & Trauma & Unexplained pain and instability & TKR with improved outcome \\
\hline 2 & 1.9 & AMOA & Lateral and PF OA & TKR but remained painful at 1 year \\
\hline 3 & 3.7 & AMOA extended & Lateral OA & TKR with poor result at 2.5 years \\
\hline 4 & 4.4 & AMOA extended & Unexplained pain & Unknown \\
\hline 5 & 4.7 & AMOA & Lateral OA & TKR mild valgus laxity at 6 months \\
\hline
\end{tabular}


Table 3

Life table for all 288 implants.

\begin{tabular}{|c|c|c|c|c|c|c|c|c|c|}
\hline \multirow[t]{2}{*}{ Year } & \multirow[t]{2}{*}{$\mathrm{n}$ at start } & \multicolumn{3}{|c|}{ n withdrawn } & \multirow[t]{2}{*}{$\mathrm{n}$ at risk } & \multirow[t]{2}{*}{$\mathrm{n}$ revised } & \multirow[t]{2}{*}{ Failure } & \multirow[t]{2}{*}{ Success } & \multirow[t]{2}{*}{ Survival (95\% CI) } \\
\hline & & Alive & Dead & Lost to follow-up & & & & & \\
\hline $0-1$ & 288 & 0 & 0 & 1 & 287.5 & 0 & 0.0 & 1.0 & 100 (100 to 100$)$ \\
\hline $1-2$ & 287 & 0 & 6 & 0 & 284 & 2 & 0.007 & 0.993 & 99.3 (98.3 to 100$)$ \\
\hline $2-3$ & 279 & 2 & 1 & 0 & 277.5 & 0 & 0.0 & 1.0 & 99.3 (98.3 to 100$)$ \\
\hline $3-4$ & 276 & 58 & 3 & 0 & 245.5 & 1 & 0.004 & 0.996 & $98.9(97.5$ to 100$)$ \\
\hline $4-5$ & 214 & 58 & 2 & 0 & 184 & 2 & 0.011 & 0.989 & 97.8 (95.7 to 99.8$)$ \\
\hline $5-6$ & 152 & 52 & 5 & 0 & 123.5 & 0 & 0.0 & 1.0 & 97.8 (95.1 to 99.8$)$ \\
\hline $6-7$ & 95 & 44 & 3 & 0 & 71.5 & 0 & 0.0 & 1.0 & 97.8 (94.1 to 99.8$)$ \\
\hline $7-8$ & 48 & 38 & 1 & 0 & 28.5 & 0 & 0.0 & 1.0 & 97.8 (89.9 to 99.8$)$ \\
\hline $8-9$ & 9 & 7 & 0 & 0 & 5.5 & 0 & 0.0 & 1.0 & 97.8 (83.9 to 99.8$)$ \\
\hline $9-10$ & 2 & 2 & 0 & 0 & 1 & 0 & 0.0 & 1.0 & 97.8 (75.1 to 99.8$)$ \\
\hline
\end{tabular}

Oxford knee as it is more securely fixed, there may well have been improvements in our selection of patients and surgical technique since the 1990s, both of which are known to be critical for the success of the Oxford knee [14].

Even though the revision rate was higher in patients who did not satisfy the strict criteria for AMOA, the survivors did not have poorer clinical outcomes (OKS, AKS-F, Tegner). This is in keeping with the results from the originating centre where a subgroup of patients with other indications also did well generally [1]. The number of patients in each subgroup with "other indications" is too small to make specific recommendations. Further work is needed, perhaps by combining the data from several centres.

The physician-assessed AKS-F score fell a little from the two years measurement to final follow-up, which is what one would expect as patients age. However, the patient-assessed Oxford Knee Score of 41 at two years was maintained at follow-up, showing sustained benefit in the patients' eyes with no suggestion of deterioration of the knee itself with time. This too is in keeping with the findings of the originating centre with the single peg version, at least to the seven years point [1].

A poorer outcome for the Oxford knee at two years in female patients was noted in a previous study [7] and was again observed here

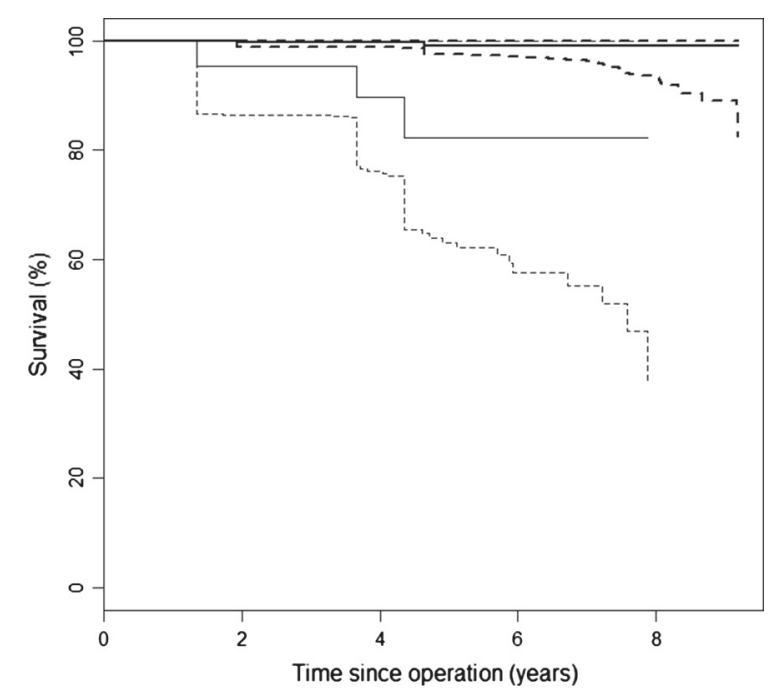

\begin{tabular}{|c|c|c|c|c|c|}
\hline \multicolumn{5}{|l|}{ AMOA } & \\
\hline At risk: & 267 & 260 & 200 & 91 & 9 \\
\hline Revised: & & 1 & 0 & 1 & 0 \\
\hline \multicolumn{6}{|l|}{ Other indic. } \\
\hline At risk: & 21 & 19 & 14 & 4 & 0 \\
\hline Revised: & & 1 & 1 & 1 & \\
\hline
\end{tabular}

Fig. 4. Implant survival curves for AMOA and extended indication cases treated with unicondylar Oxford knee prosthesis. Thick lines represent AMOA cases, thin lines other indication cases. Thick and thin dashed lines represent 95\% confidence intervals for each group, taking into account number currently at risk. The upper $95 \%$ confidence limits of the two groups overlap and form the top thick dashed line. at a longer period of follow-up. This finding is consistent with data from the NJR about knee replacements in general [4]. This does not make the cemented twin-peg option unsuitable for female patients, because the benefit in terms of an increase in OKS is similar in both groups, with no significant difference between them. Moreover, the clinical outcome in this series for female patients is better than that described for either gender in the NJR for total knee replacements. Anatomical differences between male and female distal femurs, leading to a poorer fit of knee implants in female patients, may explain the poorer outcome in female patients [15]. However, attempts to address this poorer fit by providing gender-specific implants have failed to reduce the gendergap in outcomes, leading to questions on the importance of these anatomical differences [15].

During the period of follow-up no cases of femoral loosening were found. This is encouraging as in the past we have had issues of loosening with the single peg version [5]. It is interesting that other unicompartmental knee manufacturers also provide two peg femoral components, for example the PFC Sigma partial knee (DePuy, Leeds, UK) and the Uniglide unicompartmental meniscal knee replacement (Corin, Cirencester, UK). The cementless version of the Oxford partial knee also has this design feature. This would suggest that convergent design is occurring, recognising the enhanced fixation of a two peg compared to a single peg femoral component for unicompartmental knee replacement.

Patients were in general satisfied with the results of their knee replacement in the long term, with $96 \%$ of patients very pleased or fairly pleased with the result. Still, four percent of patients were not very pleased or were very disappointed. Again, this is no worse than the results of the cemented phase III knee reported by Pandit [1] using exactly the same methodology of questionnaire. The satisfaction rate in the current study is better than after total knee replacement where the figure is only $82 \%$ in the NJR and $81 \%$ in the Swedish registry [16,17]. It is likely that the improved range of motion and preservation of the stabilizing cruciate mechanism are factors which favour unicompartmental knee replacements over total knee replacements. This is reflected by the more normal gait at faster walking speeds of patients with unicompartmental knees compared to those with total knee replacements [18].

No cases of deep infection were found in this period, which is consistent with the literature on unicompartmental devices being associated with lower infection risk than total knee replacements. It is likely that the lesser degree of dissection and operative trauma to the soft tissues causes less bleeding and haematoma formation which could provide a breeding ground for bacteria. Indeed, it is exceedingly rare for patients to require a blood transfusion in our experience and that of others [14].

The main weakness of this study is the lack of a contemporaneous control group of patients because we stopped using the single peg version in 2003 as a result of our observation of loosening [5,6]. During the last 10 years, as mentioned earlier, we have no doubt gained a better understanding of technical factors during surgery which may well have contributed to improved results. Another weakness is our lack of long- 
Table 4

Pre-operative, two-years postoperative and latest clinical outcomes for male and female patients.

\begin{tabular}{|c|c|c|c|c|c|c|}
\hline \multirow[t]{2}{*}{ Score } & \multirow[t]{2}{*}{ Gender } & \multicolumn{3}{|c|}{ Score (mean, SD) } & \multicolumn{2}{|c|}{$\begin{array}{l}\text { Score difference } \\
\text { (mean, 95\% CI) }\end{array}$} \\
\hline & & Pre-op & Two-years & Latest & Pre vs post & Post vs latest \\
\hline \multirow[t]{3}{*}{ OKS } & Combined & $22.1(8.2)$ & $40.8(7.4)$ & $41.0(8.5)$ & 19.0 & 0.22 \\
\hline & Female & $21.0(7.5)$ & $39.2(7.4)$ & $39.1(9.7)$ & (17.4 to 20.7 ) & $(-0.69$ to 1.14$)$ \\
\hline & Male & $23.7(9.1)$ & $42.4(7.2)$ & $42.9(6.7)$ & & \\
\hline \multirow[t]{3}{*}{ AKS-F } & Combined & & $84.2(18.7)$ & $80.4(20.2)$ & & -3.7 \\
\hline & Female & - & $80.0(19.0)$ & 76.7 (21.9) & & $(-5.9$ to 1.5$)$ \\
\hline & Male & & $88.2(17.5)$ & $83.9(17.9)$ & & \\
\hline \multirow[t]{3}{*}{ Tegner } & Combined & & & $2.4(1.3)$ & & \\
\hline & Female & & & $2.0(1.1)$ & & \\
\hline & Male & & & $2.8(1.4)$ & & \\
\hline \multirow[t]{3}{*}{ ROM } & Combined & & $129(8.3)$ & & & \\
\hline & Female & & $128(9.0)$ & & & \\
\hline & Male & & $131(7.2)$ & & & \\
\hline
\end{tabular}

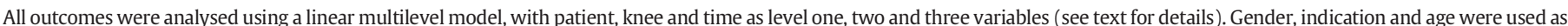

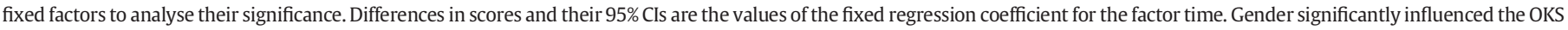

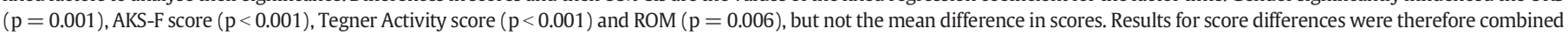

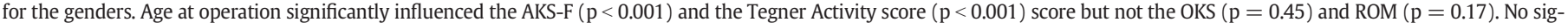
nificant influence of indication on any clinical outcome was found ( $\mathrm{p}>0.1$ for all analyses).

term follow-up radiographs although all patients were X-rayed at two years after surgery and there was no loosening around the central peg at that stage or on any subsequent radiographs carried out for any clinical reason. A strength of this study is the very complete follow-up, with only one patient whose outcome is unknown. Furthermore, the conclusions drawn from the revision data are reinforced by data from the NJR which shows our revision rate for unicondylar knees to be an "underlier" with a standardised revision ratio of 0.36 , below the lower $99.8 \%$ confidence limit for the expected ratio at the time of writing (4 Feb 2015).

\section{Conclusion}

The survival rate of the cemented twin-peg Oxford partial knee in the current series was higher than that of the single peg knee at our centre, higher than the national results for the Oxford partial knee reported by the National Joint Registry, which mainly represent outcomes of the single-peg implant, and appeared no worse than the results of the single peg knee in the hands of the originating centre. The twin-peg implant can offer secure femoral fixation, and a high degree of sustained clinical benefit and patient satisfaction.

\section{Conflict of interest statement}

None of the authors of this work have any conflict of interest to declare.

There was no financial support provided for the study apart from the senior author's charitable research fund held in the Institute of Orthopaedics of The Robert Jones and Agnes Hunt Orthopaedic Hospital, Oswestry.

\section{References}

[1] Pandit H, Jenkins C, Gill HS, Barker K, Dodd CA, Murray DW. Minimally invasive Oxford phase 3 unicompartmental knee replacement: results of 1000 cases. J Bone Joint Surg [Br] 2011;93:198-204.
[2] Svärd UC, Price AJ. Oxford medial unicompartmental knee arthroplasty: a survival analysis of an independent series. J Bone Joint Surg [Br] 2001;83-B:191-4.

[3] Wynn Jones H, Chan W, Harrison T, Smith TO, Masonda P, Walton NP. Revision of medial Oxford unicompartmental knee replacement to a total knee replacement: similar to a primary? Knee 2012;19:339-43.

[4] National Joint Registry for England, Wales and Northern Ireland. 10th Annual Report 2013. http://www/njrcentre.org.uk.

[5] Weale AE, Halabi OA, Jones PW, White SH. Perception of outcomes after unicompartmental and total knee replacement. Clin Orthop Relat Res 2001;382: 143-53.

[6] Luscombe KL, Lim J, Jones PW, White SH. Minimal invasive Oxford medial unicompartmental knee arthroplasty: a note of caution. Int Orthop 2007;31:321-4.

[7] White SH, Roberts S, Jones PW. The twin peg Oxford partial knee replacement: the first 100 cases. Knee 2012;19:36-40.

[8] White SH, Ludkowski PF, Goodfellow JW. Anteromedial osteoarthritis of the knee. J Bone Joint Surg Br 1991;73-B:582-6.

[9] Dawson J, Fitzpatrick R, Murray D, Carr A. Questionnaire on the perceptions of patients about total knee replacement. J Bone Joint Surg [Br] 1998;80B:63-9.

[10] Insall JN, Dorr LD, Scott RD, Scott WN. Rationale of the Knee Society clinical rating system. Clin Orthop 1989;248:13-4.

[11] Tegner Y, Lysholm J. Rating systems in the evaluation of knee ligament injuries. Clin Orthop 1985;198:43-9.

[12] Klein JP, Moeschberger ML. Survival analysis. Techniques for censored and truncated data. New York: Springer; 1997[etc.].

[13] Price AJ, Svard U. A second decade lifetable survival analysis of the Oxford unicompartmental knee arthroplasty. Clin Orthop Relat Res 2011 Jan;469(1):174-9.

[14] Goodfellow J, O'Connor J, Dodd C, Murray D. Unicompartmental arthroplasty with the Oxford knee. Oxford University Press; 2011.

[15] Cheng T, Zhu C, Wang J, Cheng M, Peng X, Wang Q et al. No clinical benefit of gender-specific total knee arthroplasty: a systematic review and meta-analysis of 6 randomized controlled trials. Acta Orthop 2014;85:415-21.

[16] Baker PN, van der Muelen JH, Lewsey J, Gregg PJ. The role of pain and function in determining patient satisfaction after total knee replacement: data from the National Joint Registry for England and Wales. J Bone Joint Surg Br 2007;89-B:893-900.

[17] Robertsson O, Dunbar M, Pehrsson T, Knutson K, Lidgren L. Patient satisfaction after knee arthroplasty: a report of 27,372 knees operated on between 1981 and 1995 in Sweden. Acta Orthop Scand 2000;71:262-7.

[18] Wiik AV, Manning V, Strachan RK, Amis AA, Cobb JP. Unicompartmental knee arthroplasty enables near normal gait at higher speeds, unlike total knee arthroplasty. J Arthroplasty 2013;28(9 Suppl.):176-8. 